\title{
DE LA RECLAMACIÓN DE LA PAZ A LA PARTICIPACIÓN EN LAS NEGOCIACIONES. EL FEMINISMO PACIFISTA
}

\author{
CARMEN MAGALLÓN PORTOLÉS \\ Fundación Seminario de Investigación para la Paz de Zaragoza
}

\section{EL FEMINISMO PACIFISTA, UNA TRADICIÓN NO EXENTA DE POLÉMICA ${ }^{1}$}

Las iniciativas a favor de la paz protagonizadas por mujeres se enmarcan en una corriente de feminismo internacionalista, opuesto a la guerra y la violencia, que es importante conocer y transmitir. Constituyen un legado histórico que no surge por casualidad, sino como consecuencia del lugar específico que la cultura asignó a las mujeres, fuera del ámbito público, marginadas de las decisiones sobre la paz y la guerra y excluidas de los cuerpos armados. Al mismo tiempo, las relaciones entre feminismo y pacifismo siempre fueron polémicas, por ser el pacifismo un valor que la tradición patriarcal dio por 'natural' en las mujeres y que, por tanto, como el grueso de esa tradición, pasaba a ser objeto de crítica y rechazo.

A principios del siglo XX, en la época victoriana, la igualdad entre naturaleza femenina y virtudes pacíficas era un lugar común. Las sufragistas lo utilizaron en su lucha por el voto, que no dudaban sería un voto hacia la paz. Sin embargo, voces pacifistas bien reconocidas, como la de Bertha Von Suttner, primera mujer que recibió el Premio Nobel de la Paz, en 1905, se oponían ya a esta visión:

"Alguna gente piensa que las mujeres son hostiles a la guerra por naturaleza. Están en un error. Sólo las mujeres progresistas, aquellas que han sido capaces de educarse a sí mismas en una conciencia social, que han tenido la fuerza de no dejarse fascinar por instituciones con centenares de años, encuentran también la energía para oponerse a ellas»².

1. Para una visión amplia de este tema, véase: MAGALLón, Carmen: Mujeres en pie de paz, Madrid, Siglo XXI, 2006.

2. Discurso al movimiento de Mujeres por la Paz alemanas, 1914 (OldFIELD, Sybil: Women Against the Iron Fit. Alternatives to Militarism (1900-1989), Cambridge, Basil Blackwell, 1989, p. 210). 
Una muestra de la capacidad creativa de las sufragistas, a favor de la paz, fue la iniciativa de convocar en La Haya, en plena I Guerra Mundial, el Primer Congreso Internacional de Mujeres. En medio de la contienda, cuando ya habían transcurrido nueve meses de guerra, la realización de este congreso fue todo un hito. En primer lugar, porque las mujeres mostraron su capacidad de unirse por encima del enfrentamiento armado entre sus países; también porque en él se sentaron las bases de un movimiento internacional de mujeres por la paz. Bajo la presidencia de Jane Addams, reformadora social norteamericana, sufragista y antimilitarista, que recibiría el Premio Nobel de la Paz en 1931, alrededor de un millar de mujeres (1.136 mujeres con voto y más de 300 visitantes y observadoras) en representación de unas 150 organizaciones de 12 países, beligerantes y neutrales, se reunieron para elaborar una estrategia de paz, protestar contra la locura de la guerra y hacer un llamamiento a la mediación inmediata de los países neutrales. Mujeres de distintas tendencias apoyaron el Congreso: laboristas británicas, sufragistas y sindicalistas de distintos países, mujeres de organizaciones tan diversas como las Trabajadoras Agrícolas de Hungría, la Liga para la protección de los Intereses de los Niños de Holanda o la Asociación de Mujeres Abogadas de Estados Unidos ${ }^{3}$.

El Congreso de la Haya ${ }^{4}$ fue un hijo de la Alianza Internacional por el Voto de la Mujer, aunque no recibió el apoyo oficial de todas las organizaciones que la conformaban. Debido a la guerra, la Alianza había suspendido una reunión que iba a celebrarse en junio de ese mismo año en Berlín. Aletta Jacobs, presidenta de la Alianza sufragista holandesa y una convencida de que las mujeres podían jugar un importante papel contra la guerra, junto a Kathleen Courtney, secretaria honoraria de la Unión Nacional de Sociedades por el Sufragio Femenino, Chrystall MacMillan, abogada escocesa, sufragista y pacifista, y Catherine Marshall, decidirían convocarlo. Impacta pensar en la fuerza de estas mujeres de principios de siglo, dispuestas a actuar en medio de todas las dificultades de una guerra para tratar de pararla.

En La Haya surgió el Comité Internacional de Mujeres para una Paz permanente. En el siguiente congreso, celebrado en Zurich, en 1919, se crearía la Liga Internacional de Mujeres por la Paz y la Libertad (Women's International League for Peace and Freedom, WILPF), una organización con pretensiones de unir dos movimientos que las asistentes sentían vitalmente vinculados: el movimiento feminista y el movimiento pacifista. El Comité Ejecutivo de WILPF fue elegido sobre bases individuales, no nacionales y, para ejercer su influencia, se estableció en Ginebra, la ciudad que albergaba las oficinas principales de la Liga de las Naciones.

Las fundadoras de la Liga Internacional de Mujeres por la Paz y la Libertad eran mujeres de clase media, con formación académica, algunas de ellas gradua-

3. NASH, Mary: Mujeres en el mundo. Historia, retos y movimientos, Madrid, Alianza, 2004.

4. Sobre el Congreso de La Haya, puede verse BUSSEY, Gertrude y TIMS, Margaret: Pioneers for Peace.

Women's International League for Peace and Freedom 1915-1965, Oxford, Alden Press, 1980. 
das en Oxford o Cambridge, que hablaban varios idiomas y no se arredraban ante viajes y dificultades. Se orientaban hacia una visión del feminismo que no excluía ningún asunto en la búsqueda de la participación política plena para las mujeres. Era un feminismo transnacional, que se interesaba por la economía y las relaciones internacionales y que creía que los conflictos internacionales tenían que afrontarse de un modo civilizado. No eran «un conjunto de mujeres socializadas para la subordinación amable, reaccionando con el disgusto apropiado a los horrores de la guerra, ansiosas de cumplir el rol maternal de aplicar vendas a las heridas que los muchachos se habían infligido unos a otros» ${ }^{5}$. Creían realmente en la posibilidad de solucionar las disputas de otro modo, a través de un arbitraje internacional, querían influir en la marcha de los acontecimientos y no estaban dispuestas a tener un papel subordinado. Con su iniciativa no marginaban los derechos de las mujeres, porque "para ellas la cuestión de la guerra y la paz era una preocupación feminista, un desarrollo lógico de su comprensión de lo que significaba un rol de igualdad, pleno, de las mujeres» ${ }^{6}$.

Las mujeres socialistas europeas de la época también creían que su compromiso socialista era a favor de la paz, pensando que los trabajadores del mundo comprometidos con el socialismo nunca tomarían las armas unos contra otros. En Inglaterra una de las mayores campañas de las mujeres trabajadoras fue la Cruzada de las Mujeres por la Paz, iniciada a principios de la guerra y que tuvo su auge en 1917-18. Las socialistas Agnes Dollan, de Glasgow y Helen Crawford, fundadora de la Liga Internacional de Mujeres en Escocia, fueron sus principales impulsoras. Hubo concentraciones y mítines, no exentos de tensiones en muchas ciudades, pues en algunos casos los himnos patrióticos impidieron hablar a las oradoras ${ }^{7}$. Líderes socialistas destacadas como Clara Zetkin y Rosa Luxemburg pasaron los años de la guerra entrando y saliendo de la prisión, por denunciar la guerra como imperialista. Pero, de modo análogo a lo que sucedió con el internacionalismo del movimiento de mujeres sufragistas, la Primera Guerra Mundial dividiría al movimiento socialista.

Tras la experiencia de la Primera Guerra Mundial, fue más difícil mantener que las mujeres eran una fuerza de paz, tal como pensaban inicialmente las feministas. Lo cierto es que la guerra dividió al sufragismo, expresión del feminismo del momento. Muchas sufragistas dedicaron sus esfuerzos a incitar a los varones a alistarse voluntariamente y a lograr que las mujeres se incorporasen a los trabajos abiertos a ellas en apoyo a la guerra, por ejemplo, fabricar las balas que mataban a los hombres.

5. Vellacot, Jo: «A Place for Pacifism and Transnationalism in Feminist Theory: the early work of the Women's International League for Peace and Freedom", Women's History Review, 2: 1 (1993), p. 39.

6. Ibíd., pp. 28-29.

7. IDINGTON, Jill: «La campaña de las mujeres por la paz. Historia de una lucha olvidada», en Dorothy Thompson (comp.): Over our dead bodies, Londres, Virago, 1983 (Trad. Mireia Bofill, Antes muertas. Mujeres contra el peligro nuclear, Barcelona, LaSal, 1984, p. 202). 
No obstante, la perspectiva y la capacidad de iniciativa de las mujeres por la paz reaparece constantemente. Gandhi mantuvo que había aprendido las técnicas de la no-violencia y de la desobediencia civil de las mujeres, principalmente de las sufragistas británicas. Y es que en el movimiento por el voto, las sufragistas desarrollaron formas de presión y acción pública distintas a las que se conocían en su tiempo, métodos no-violentos. Seguramente por esto, por el origen femenino de estas prácticas, las mujeres fueron entusiastas seguidoras de las propuestas gandhianas. Es un hecho que más del $60 \%$ de los integrantes de la marcha de la Sal, que tuvo lugar en marzo de 1930 en la India, fueron mujeres ${ }^{8}$.

En el transcurso de la Segunda Guerra Mundial, las mujeres formaron parte de la Resistencia y algunas figuras destacadas como Simone Weil, en Francia, Hellen Keller en los EEUU o Sophie Schöll en Alemania, que habían trabajado activamente contra la idea de la guerra, renunciaron a su pacifismo, y pasaron a colaborar en la lucha contra el nazismo. En esto coincidirían con la opción tomada por algunos hombres pacifistas.

\subsection{La invitación a no repetir los errores de los hombres: Virginia Woolf}

Durante la guerra civil española algunas fotografías enviadas por el Gobierno español a Inglaterra en las que se reflejaban los horrores de la guerra fueron a parar a manos de Virginia Woolf, la escritora del grupo de Bloomsbury. Se le pedía que expresara su opinión sobre cómo podían las mujeres ayudar a evitar la guerra. Con este motivo escribió Tres Guineas, publicado inicialmente en 1938, libro que acoge una de las reflexiones más lúcidas y profundas que se han hecho sobre los aspectos de género implicados en el problema. En él, V. Woolf hace una invitación a la actitud crítica ante las instituciones creadas por los varones excluyendo a las féminas, y una exhortación al desarrollo de la creatividad de las mujeres para pensar el mundo desde sí mismas. A la pregunta de cómo pueden las mujeres colaborar a evitar la guerra, escribirá: "la mejor manera en que podemos ayudarle a evitar la guerra no consiste en repetir sus palabras y en seguir sus métodos, sino en hallar nuevas palabras y crear nuevos métodos»".

Para ella la prevención de la guerra requeriría el desmantelamiento del sistema patriarcal de sexo-género: la ruptura de la división entre esferas de varones y esferas de mujeres, y la despolarización de la masculinidad y la feminidad. Porque no sólo la masculinidad con su contenido de agresiva afirmación contribuye a la guerra, también las mujeres son responsables al admitir y fomentar estas posturas. Los varones, dice, han sido socializados en la creencia de que es mejor matar que morir y que su virilidad depende del éxito alcanzado en dominar. Las mujeres, a su vez, son socializadas para aceptar la dominación. Creía, sin embargo, que por razones histórico-sociales, las mujeres tenían una mayor

\footnotetext{
8. De entre las treinta mil personas arrestadas en esta acción, diecisiete mil eran mujeres (BROCKUTne, Birgit: Educating for Peace. A Feminist Perspective, Oxford, Pergamon Press, 1985).

9. Woolf, Virginia: Tres Guineas, Barcelona, Lumen, 1977 (1938), p. 193.
} 
potencial para oponerse a la guerra, debido a su histórica exclusión del poder y la riqueza. Para ella esa era la razón de que el patriotismo, tantas veces esgrimido para pelear, no tuviera sentido para las mujeres. Por el contrario, escribió: «en mi condición de mujer no tengo patria. En mi condición de mujer, no quiero tener patria. En mi condición de mujer, mi patria es el mundo entero» ${ }^{10}$.

Elena Grau, del grupo de Mujeres de En Pie de paz, al hilo de sus reflexiones sobre Tres Guineas, escribe:

«La singularidad de las mujeres con respecto a la guerra no es que ellas sean pacíficas por naturaleza biológica o social, sino que son ajenas al orden sociosimbólico que la sustenta (...) Virginia habla muy poco de la experiencia, de las consecuencias y del horror de la guerra porque parte de una idea, nunca la guerra, y no necesita argumentarla (...) y al poner la guerra como medida, o como horizonte de nuestra acción, trasciende la idea de guerra como hecho bélico y se interesa por todo aquello que en nuestro hacer apunta en última instancia a sostener unas relaciones, una cultura y un mundo simbólico que albergan la violencia y conducen a la guerra $»^{11}$.

Inscrita en esta corriente de pensamiento, Luisa Muraro recupera, al hilo de la escritura de Simone Weil sobre la guerra de Troya, la autoridad y la independencia simbólica de un hilo que corre, dice, desde la Antigüedad hasta nuestros días. Comparte con Weil que la raíz de lo absurdo de la historia humana, nace de otro absurdo: la naturaleza del poder. Destaca que para Weil, frente al mecanismo del poder que se expande sin límite,

«es necesario oponer la inteligencia que sólo el sentido de nuestra relatividad puede darnos. El mal simbólico de la guerra (y del poder) reside prácticamente en la destrucción de esta inteligencia. O peor, en hacerla impracticable, porque cuando hay una guerra todo gira en torno al círculo vicioso de un prestigio que no puede negociar con el adversario, so pena de la propia extinción (...) Es la política lo que interrumpe el mecanismo de las relaciones de fuerza tanto en este mundo como en nuestras almas. Política es una brecha de libertad en los mecanismos ciegos del poder/carencia de poder, y éste fue el sentido profundo y constante de su [de Weil] pacifismo: obstaculizar el paso a la guerra para hacer sitio a los conflictos políticos $\aleph^{12}$.

Hacer visible el protagonismo de las mujeres en los movimientos de paz no busca ensalzarlas o defender una excelencia moral femenina, dejando fuera, en el reino de las tinieblas de la fuerza bruta, a los varones. No equivale a decir que ellas son mejores ni peores que ellos. Desde una posición de rechazo del determinismo biológico, hay que afirmar que para hombres y mujeres, la paz es una opción, libre en la medida en que unas y otros podemos rebelarnos frente al exterior que trata de constituirnos según viejos esquemas heredados. Es una opción enmarcada, eso sí, en el horizonte de un conflicto identitario, en medio del desasosiego que provoca el reto de criticar lo que no nos gusta de

10. Ibíd., p. 148.

11. CRAU, Elena: "Sentada en mi lado del abismo. Sobre Tres Guineas de Virginia Woolf», En Pie de Paz, 52 (2000), p. 43.

12. MURARO, Luisa: «Si la política vence a la guerra», L'Unitá, viernes 4 de Junio de 1999. 
la identidad impuesta y la afirmación de lo que cada uno y cada una somos o queremos ser.

Un reto permanente, para varones y mujeres, es seguir discerniendo el significado de ser diferentes, de que haya dos sexos en el mundo, pues como escriben las mujeres del grupo Giulia Adinolfi, hasta ahora nos hemos limitado en el mejor de los casos a pensar en un neutro abarcador -que esconde a uno de los sexos-y en el peor a una definición impuesta y forzada sobre el otro -el eterno femenino, que eterniza la subordinación-. En su empeño de encontrar un sentido al ser mujer, Elena Grau, que junto a Isabel Ribera y Violeta Ibáñez conforman el citado grupo, rechaza considerarlo algo irrelevante ${ }^{13}$, y comparte lo que Giulia Adinolfi escribió hace más de veinticinco años:

"Las mujeres tendrían que ser capaces de asumir crítica y libremente su propia tradición, de medirse con ella, de rechazar sus elementos negativos y de reivindicar, en cambio, aquellos otros que -cualquiera que haya sido su función- revelan hoy una potencialidad positiva» ${ }^{14}$.

\subsection{Mujeres que se organizan para construir la paz}

En la segunda mitad del siglo XX, el protagonismo de las mujeres en el movimiento por la paz, sobre todo en el pacifismo nuclear, es innegable. Todavía quedan cerca las iniciativas que desplegaron grupos de mujeres de toda Europa contra la instalación de misiles nucleares en los años 80. Las mujeres llevaron a cabo marchas sobre Paris, en 1981; Minsk, 1982 y Washington, 1983, y durante años mantuvieron iniciativas como el campamento de Greenham Common, en las inmediaciones de una base de misiles en Gran Bretaña. Greenham Common llegó a ser una fuente de inspiración para el conjunto del movimiento por la paz. El trabajo en pequeños grupos constituidos por afinidad, las decisiones tomadas por consenso, la firme decisión de unir los fines y los medios y el enfoque de convertir la vulnerabilidad en fuerza, pasó a ser una filosofía que enriqueció el legado histórico de la no-violencia.

Surgieron también los grupos de Mujeres de Negro, extendidos hoy por un gran número de países en el mundo, que se afirman feministas; las Madres y abuela de Plaza de Mayo; la Coordinadora de Viudas de Guatemala (CONAVIGUA); la Coordinadora de Madres del Salvador (COMADRES), la Ruta Pacífica de las Mujeres Colombianas; Jerusalem Link, en Palestina-Israel; grupos en Irlanda, en Chipre, en Liberia, en Somalia...

La implicación femenina en la construcción de la paz se plasma en el trabajo de base que llevan a cabo grupos de mujeres extendidos por todo el mundo. Las filosofías y los objetivos que les guían son diversos, aunque en general comparten el intento de deslegitimar la lógica que pone en juego la vida de los seres

13. GRAU BiosCA, Elena: «1980-2005, veinticinco años sin Giulia y con Giulia», Mientras Tanto, 94 (2005), pp. 39-44.

14. ADINOLFI, Giulia: «Sobre las contradicciones del feminismo», Mientras Tanto, 94 (2005), pp. 8385, citado por GRAU BIOSCA, Elena: «1980-2005..., op. cit., p. 41. 
humanos para perseguir intereses materiales, ideológicos, de poder o de soberanía. Hay grupos de mujeres organizados de muchos tipos: para oponerse a la guerra o las políticas militaristas y de agresión que llevan a cabo sus gobiernos o sus grupos de pertenencia; para acercar, a través de la relación y la búsqueda de puntos comunes, a personas de grupos enfrentados, de los que ellas forman parte; para la búsqueda de soluciones no militares a conflictos estructurales; contra la impunidad: para que no se repitan los genocidios, las desapariciones y las persecuciones sufridas por determinados grupos humanos; para apoyar a mujeres que viven en situaciones de guerra o de falta de libertad y derechos humanos, en países distintos al suyo, y para lograr que el trabajo de base de las mujeres cuente en la toma de decisiones (trabajo de lobby, por ejemplo el que lleva a cabo UNIFEM, algunas parlamentarias del Parlamento Europeo, y grupos y mujeres de EEUU, como CODEPINK).

\section{LAS MUJERES Y LA PAZ: UNIDAS SIMBÓLICAMENTE Y EXCLUIDAS DE LA TRADICIÓN POLÍTICA}

La unión simbólica entre mujeres y paz fue acompañada de la exclusión de ambas del ámbito de la política. La tradición que excluye a las mujeres es la misma tradición política que excluye a la paz: Maquiavelo, Clausewitz y la inevitabilidad de la guerra, Bismark y la realpolitik. Todavía hoy la política internacional considerada realista, práctica y patriota, se asienta sobre el derecho a la guerra, y esta cercanía simbólica entre paz y mujeres constituye una fuente de resistencias a la universalización del valor de la paz cuya asociación con lo femenino equivale en la práctica a una devaluación. La ligazón mujerespaz se mantiene de modo persistente pese a chocar con abundantes datos de la realidad, tanto de la historia pasada como de la más reciente. Éstos ponen de manifiesto la participación y el apoyo de las mujeres a la guerra, en formas muy variadas: ya sea como combatientes en los distintos grupos armados, ya sea sosteniendo la producción de la munición, ya dando apoyo logístico a los contendientes o del modo particularmente específico señalado por Virginia Woolf, en Tres Guineas: admirando a los héroes.

El determinismo biológico implícito en esta caracterización dicotómica ha sido ampliamente criticado desde distintas disciplinas científicas por las corrientes de pensamiento feministas. La afirmación de Simone de Beauvoir de que la mujer no nace sino que se hace puede hacerse extensiva al varón. Pues los varones también fueron naturalizados y debido a su sexo obligados a hacer el servicio de armas. La diferencia es que esta característica natural, su sexovarón que lo liga a la fuerza le concede una posición de poder que se niega a las mujeres.

El mantenimiento de la dicotomía mujer pacífica-hombre violento no favorece el avance de una cultura de paz, sino que lleva en sí el germen del mantenimiento del actual estado de cosas y de relaciones en el mundo. La crítica de esta dicotomía efectuada por los estudios feministas está aportando argumentos para desvincular el valor de la paz de un estereotipo. Los análisis aportados por los estudios feministas, en particular la profundización en el carácter y mecanis- 
mos de exclusión femenina, permiten comprender las resistencias a la paz que derivan de los avatares de una exclusión compartida. De modo similar, los argumentos, teorías y movimientos sociales que persiguen la inclusión de las mujeres en la comunidad política, y la transformación de la política y la formulación de los derechos a la luz de su experiencia, están sirviendo y contribuyendo a la inclusión del valor de la paz en la comunidad de los derechos ${ }^{15}$.

La negación de una predisposición natural de las mujeres hacia la paz, no equivale a negar su opción de constituirse en sujeto colectivo de construcción de paz. Un sujeto que, precisamente por su tradicional marginación política, puede ser percibido como ajeno a la influencia de los actores más polarizados en el conflicto. A menudo, las iniciativas para hacer la paz que vienen de parte de las mujeres merecen a la comunidad una mayor confianza que aquéllas que provienen de la élite política. Ya Virginia Woolf escribió que las mujeres, excluidas en su tiempo de los derechos de igualdad política, pertenecían en verdad a otra sociedad, a la Sociedad de las Extrañas ${ }^{16}$, al ser extrañas al orden social establecido. Esta extrañeza todavía se arrastra hoy, aunque sea en el plano simbólico. Se arrastra como un déficit que limita y también como una capacidad que posibilita a las mujeres optar por prácticas creativas de actuación social. Actuar desde la exclusión encierra un enorme potencial de cambio. Como extrañas a las estructuras políticas patriarcales, las mujeres tienen la libertad de proponer y llevar a cabo soluciones innovadoras ante los conflictos. Pueden buscar sus propias palabras y tratar de no transitar por los errores de los varones. Es lo que hacen muchos grupos de mujeres por la paz: desarrollar iniciativas enfocando el problema desde una lógica y una perspectiva radicalmente diferente.

\section{MUJERES EN LOS PROCESOS DE PAZ}

El enorme trabajo por la paz que llevan a cabo las mujeres no tiene su correlato en el nivel de la toma de decisiones. La tarea de construir la paz habría de recaer en el conjunto de la sociedad, pero al igual que sucede con las decisiones sobre el inicio de la guerra o la responsabilidad de las hostilidades, los avances o estancamientos en el proceso de negociar la paz se encuentran predominantemente en manos de los hombres. El protagonismo de las mujeres en las organizaciones civiles y de base, no es fácilmente trasladable a la mesa de negociaciones. Existen resistencias por todas partes: por la inercia del poder establecido, por las facciones que contienden y por las propias mujeres que a menudo no quieren sentarse con los líderes responsables de los crímenes de ambos lados.

El principal argumento para defender la participación de las mujeres en los procesos de paz sigue siendo la equidad, el derecho que tenemos a participar,

15. MaGallón, Carmen. "El derecho humano a la paz y la sociedad civil. Una mirada desde las vidas de las mujeres», en Xesús R. Jares, Josu Ugarte, Mikel Mancisidor y Maria Oianguren (coords.): El papel de la investigación para la paz ante la violencia en el País Vasco, Bilbao, Bakeaz y Gernika Gogoratuz, 2006, pp. 117-136.

16. Wbolf, Virginia: Op. cit., p. 145. 
un derecho que es evidente pero que hay que reafirmar porque la tradición patriarcal de la mayoría de las culturas no lo ha reconocido en el pasado y aún en el presente existen resistencias a hacerlo. A lo que puede añadirse el hecho constatado de que las mujeres llevan a la mesa de negociación temas y asuntos que ningún otro actor suele llevar. Ahora bien, no basta con estar, para influir efectivamente en los acuerdos, es preciso articular agendas de consenso y una fuerza social y política que las respalde ${ }^{17}$.

El argumento que se esgrime para la exclusión femenina de la toma de decisiones es que son las partes contendientes las que han de negociar la paz, y que la presencia o ausencia de las mujeres no es relevante ${ }^{18}$. Se olvida que ellas resultan afectadas por los conflictos bélicos de un modo específico, por el papel que se les atribuye y el tipo diferencial de agresiones que sufren. También se olvida que la paz es un proceso que pertenece a las comunidades, no sólo a los líderes y que es importante que todo el conjunto social se involucre en las tres tareas a abordar tras un conflicto armado: la reinserción de los combatientes, la reconstrucción y la reconciliación. Además, frente a una visión limitada de las negociaciones de paz, en la que sólo cuentan los elementos estrictamente bélicos, hay que tener en cuenta que no se trata de un acontecimiento puntual sino de un proceso que va a marcar el futuro desarrollo de la vida del país en cuestión, ya que la paz incluye asuntos como: acuerdos para compartir el poder, para la reconstrucción económica, para la desmovilización y reintegración de los combatientes; legislación sobre derechos humanos, sobre la regulación del acceso a la tierra, a la educación y a la salud; el estatus de las personas desplazadas, el papel de la sociedad civil, etc. Es cuando pensamos en las negociaciones como un proceso, del que depende la estructura social que va a reconstruir la convivencia, cuando se ve la importancia de la participación de las mujeres en él.

En el estudio de casos realizado por Anderlini, se agrupan ejemplos de procesos de paz en distintos países ${ }^{19}$ : en Sudáfrica, Irlanda del Norte y Guatemala, la fuerza de los movimientos de mujeres fue llevada al proceso de negociación de la paz; en otros lugares como Liberia, Burundi, Georgia y Palestina, las mujeres trataron y siguen tratando de influir en la construcción de la paz, en medio de la guerra; en Camboya, las mujeres, en la fase de reconstrucción postconflicto, negociaron avances hacia la igualdad ${ }^{20}$. Puede observarse que, aunque con cierta lentitud, la tendencia es hacia una creciente inclusión de mujeres en los procesos de paz y en la mesa de negociación.

17. Luz Méndez, Presidenta del Consejo consultivo de la Unión Nacional de Mujeres Guatemaltecas (UNAMG), en su intervención en las Jornadas «Las mediaciones femeninas. Una práctica de paz", Barcelona, 2005.

18. ANDERLINI, Sanam Naraghi: Women at the Peace Table. Making a Difference, Nueva York, The United Nations Development Fund for Women (UNIFEM), 2000.

19. Ibíd.

20. Para el caso de Camboya, véase Alonso Ollacarizaueta, Lucía: Reanudar lazos rotos. Un estudio sobre reconciliación en Camboya, Barcelona, Icaria, 2003. 


\subsection{El caso de Sudáfrica}

En Sudáfrica, las mujeres involucradas en la política recibieron la fuerza del movimiento de mujeres, llegando a alcanzar altos puestos en el Congreso Nacional Africano (CNA). En los años previos a las elecciones de 1994, las activistas formaron una fuerte Coalición Nacional de mujeres que se unieron por encima de las líneas divisorias de estatus social y adscripción política. Dos organizaciones, la llamada Black Sash, integrada mayoritariamente por mujeres blancas, casadas con hombres de negocios y la Unión de Trabajadoras Domésticas, formada por mujeres negras formaron una federación anti-apartheid:

«Nunca antes se habían hablado, y ahora eran parte de una coalición en la que la presidenta era una trabajadora doméstica. Juntas, las pobres y las ricas llegaron a ser conscientes de que habían sufrido de manera similar. Se dieron cuenta de que hombres ricos y pobres pueden tratar a las mujeres del mismo modo humillante. Ahora estaban juntas por una cuestión de dignidad $»^{21}$.

La Coalición movilizó el apoyo de los grupos de base para una propuesta del CNA que planteaba que las mujeres debían estar representadas en condiciones de igualdad en las negociaciones. No estaban dispuestas a que sucediera lo que habían visto en situaciones similares, en las que las mujeres, después de haber hecho las mismas contribuciones y sacrificios que los hombres en la lucha por la liberación, habían sido apartadas de la mesa de negociación y relegadas en la sociedad emergente. Uno de los resultados de su participación en el proceso de paz fue el aumento de su presencia en las instituciones. En las primeras elecciones democráticas, en 1994, las mujeres pasaron a tener el $24 \%$ de los puestos de la Asamblea Nacional y el Senado, cuando en la época del apartheid sólo tenían el 2,8\%. Cheryl Carolus ${ }^{22}$, que en 1994 fue elegida Deputy Secretary General del Congreso Nacional Africano, dice que, además de en la sociedad civil, gran parte del proceso de negociación se dio en las estructuras del propio partido que encabezaba el cambio, en el CNA.

El proceso de paz sudafricano fue un ejemplo de participación política a todos los niveles ${ }^{23}$. Desarrolló propuestas innovadoras para afrontar el pasado y el futuro, como la Comisión de la Verdad y la Reconciliación. En esta comisión, las mujeres tuvieron un importante papel: fueron el $41 \%$ de los comisionados y aportaron el 56,5\% de los más de veinte mil testimonios presentados. Su actitud y facilidad para establecer relaciones empáticas ayudó a la creación de un clima favorable para testificar. Las estructuras separadas para las mujeres, como la Audiencia de Mujeres de Johannesburgo, proporcionó un espacio adecuado para aportar testimonios acerca de los crímenes cometidos contra las mujeres

21. ANDERLINI, Sanam Naraghi: Op. cit., p. 14.

22. En 1998, asumió el cargo de Alta Comisionada de su país en Londres (South African High Commissioner).

23. BARNES, Catherine (ed.): Haciendo propio el proceso. La participación ciudadana en los procesos de paz, Gernika-Lumo, Gernika Gogoratuz, 2004. 
De la reclamación de la paz a la participación en las negociaciones. El feminismo pacifista

en la época del apartheid y de este modo una visión más completa de lo que sucedió ${ }^{24}$.

\subsection{El caso de Irlanda del Norte}

Otro caso en el que la unión de las mujeres logró que éstas entraran a formar parte de la mesa negociadora es el de Irlanda del Norte. Durante décadas, católicas y protestantes trabajaron juntas por el diálogo y la colaboración entre las dos comunidades. En 1976, las actividades e iniciativas desplegadas por Betty Williams y Mairead Corrigan, de Mujeres Irlandesas por la Paz, les hicieron merecedoras del Premio Nobel de la Paz.

En 1996, el mediador internacional en el conflicto, Georges Mitchell, puso como condición para participar en la mesa de negociaciones que los nominados por las partes tenían que ser representantes elegidos en las urnas. Con este requisito los diez mayores partidos no tenían problema para ser incluidos pero las mujeres carecían de una opción política propia. Ante este vacío, un grupo de activistas convocó una reunión a la que asistieron más de doscientas organizaciones de mujeres de ambas comunidades. El resultado fue la creación de la Coalición de Mujeres de Irlanda del Norte (Northern Ireland Women's Coalition, NIWC). La Coalición se presentó a las elecciones y consiguió dos escaños; este resultado les aseguró un lugar en la mesa de negociaciones.

Annie Campbell, sindicalista y feminista, de origen protestante, una de las que participó en las conversaciones que condujeron a las negociaciones de Viernes Santo, en nombre de la Coalición de mujeres, explica que ellas operaron como mediadoras, insistiendo en los derechos humanos y la inclusión: todos tenían que estar en las conversaciones. Helen Jackson, parlamentaria británica que trabajó de cerca con las organizaciones de mujeres en Irlanda del Norte, declaró que las preocupaciones que ponen las mujeres sobre la mesa de negociación son, a menudo, muy diferentes a las de los hombres. Para muchas, importa más la educación y el cuidado de los hijos y la situación de su hogar que otras cuestiones.

\subsection{El caso de Somalia}

En la Conferencia Nacional por la paz de Somalia, que tuvo lugar en Arta, Djibouti, el 2 de mayo de 2000, las delegaciones venían en nombre de cuatro clanes mayoritarios y una coalición de más pequeños. En este nuevo intento por conseguir la paz que, desde 1991, hacía ya el número catorce, 92 mujeres somalíes se presentaron como el Sexto clan. La coalición de mujeres o Sexto Clan incluía mujeres de todos los clanes, unidas por la noción de igualdad de género. Cuenta Asha Hagi Elmi, líder del Sexto Clan, cómo:

«En Arta, hicimos la presentación de un buranbur -una composición poética especial cantada por mujeres- para mostrar el sufrimiento de las mujeres y los niños a

24. Bogodo-Madikizela, Pumla: Women's Contributions to South Africa's Truth and Reconciliation Commission, Women Waging Peace, The Hunt Fund, 2005. 
lo largo de 10 años de guerra civil. Hicimos lobby para lograr una cuota femenina en la futura legislatura, la Asamblea Nacional Transitoria (Transitional National Assembly, TNA). Pero encontramos la oposición de los delegados hombres. Nos dijeron que ningún hombre estaría de acuerdo en ser representado por las mujeres» ${ }^{25}$.

Finalmente, lograron establecer una Carta Nacional que establecía la protección de los derechos humanos de las mujeres, los niños y las minorías, y garantizaba que en la Asamblea Nacional Transitoria, de un total de 245 puestos, 25 serían para las mujeres. Esta Carta Nacional fue una de las mejores de la región y la mejor en el mundo musulmán²6.

\subsection{El caso de Palestina-Israel}

Las mujeres palestinas e israelíes comenzaron a reunirse y a negociar entre ellas ya desde 1988, cuando se crea el grupo de Mujeres de Negro. En los tiempos en que esas conversaciones estaban prohibidas en Palestina y eran ilegales en Israel, se reunían en secreto para hablar, en casas particulares y en iglesias. Luego se encontraron en Basilea, Berlín, Bruselas, Bolonia y otras ciudades europeas. En 1994, con el apoyo de la Comisión europea, se crean dos centros de mujeres, uno en la Jerusalén Este palestina, Jerusalem Center for Women y otro en la Oeste, israelí, Bat Shalom, que dieron lugar a Jerusalem Link. Las mujeres de los dos centros, ligadas a partidos y también al Parlamento de Israel, con amplios vínculos internacionales, desarrollaron a lo largo de los años un diálogo constante, que se llevó a cabo sobre todo a través de cartas y declaraciones. Sumaya Farhat-Naser, palestina de los territorios ocupados y directora del Jerusalem Center for Women, ha documentado los esfuerzos y dificultades vividos por estas mujeres israelíes y palestinas en la búsqueda de una paz justa para sus pueblos $^{27}$. Sumaya explica que fue en Bruselas, donde establecieron, en 1992, los principios políticos básicos para llevar a cabo un trabajo conjunto por la paz, y que estos principios:

«crearon un marco vinculante y nos ofrecieron orientación para el trabajo en común: igualdad y paridad de nuestros dos pueblos, el reconocimiento mutuo de los estados nacionales de Palestina e Israel, así como la confirmación de Jerusalén como ciudad abierta que pertenece a ambos y que debe ser la capital de los dos estados. Estos principios nos ofrecían protección frente a las acusaciones de traición de nuestra propia gente, al mismo tiempo que se daba también una legitimación política a nuestro trabajo y se preparaba el camino para una aproximación. Debían contribuir a la construcción de una paz segura, sin violencia y en justicia. Además de los principios políticos del diálogo, las mujeres pacifistas desarrollaron principios

25. ReHn, Elizabeth y Sirleaf, Ellen J.: Women War and Peace. The Independent Experts' Assesment on the Impact of Armed Conflict on Women and Women's Role in Peace-building, Nueva York, The United Nations Development Fund for Women (UNIFEM), 2002, p. 79.

26. Ibíd.

27. FARHAT-NASSER, Sumaya: En la tierra de los olivos. Una historia de mujeres por la paz (prólogo y traducción de Anna Tortajada), Barcelona, El Aleph, 2006. 
de comunicación, que debían servir al mismo tiempo como barandillas para poder mantener distintos equilibrios en las conversaciones» ${ }^{28}$.

En 2001, tras el comienzo de la Segunda Intifada, se rompen los programas conjuntos. En ese momento, Svirsky y Farhat-Naser, que habían sido directoras de los dos centros que conformaban Jerusalem Link, escribieron una declaración conjunta, con el título: "Nos negamos a ser enemigas». En la declaración condenan toda forma de violencia, brutalidad y terrorismo, venga de parte de grupos, gobiernos, ejércitos o personas individuales:

«Estamos hartas de muertos en ambos lados. Demasiados niños palestinos e israelíes han muerto, han quedado huérfanos o tullidos para el resto de sus vidas. Demasiados de nuestros hijos, padres y hermanos han matado. Porque la guerra no sólo convierte en víctima a los inocentes, también embrutece a los que dirigen la guerra» ${ }^{29}$.

Y tras reconocer el esfuerzo realizado por mujeres israelíes y palestinas, para mantener el diálogo, afirman que ellas sí serían capaces de encontrar una salida negociada, pues

«aunque hubo diferencias de opinión y debates, y a menudo nuestras conversaciones se celebraban en circunstancias dolorosas, siempre nos hemos mantenido fieles a una concepción común de la paz. Si dependiera de nosotras, hace tiempo que habríamos llegado a un acuerdo de paz que regulara los difíciles problemas que hay entre nuestros dos estados (...) El movimiento de mujeres pacifistas en Palestina y en Israel cree que ha llegado el momento de poner fin al derramamiento de sangre. Ha llegado el momento de rendir nuestras armas y nuestros miedos. Nos negamos a aceptar aún más guerra en nuestras vidas, en nuestras comunidades, en nuestras naciones. Nos negamos a aceptar la violencia. Nos negamos a ser enemigas» ${ }^{30}$.

\section{LA RESOLUCIÓN 1325 DEL CONSEJO DE SEGURIDAD DE LA ONU}

El apoyo de la Comunidad Internacional es muy necesario en zonas donde la voz de las mujeres es silenciada de manera permanente. Las alianzas transversales, que cruzan niveles y naciones, y circulan desde los movimientos de base a las agencias de Naciones Unidas y viceversa, pasando por la implicación personal de mujeres de reconocido prestigio, pasan a ser decisivas. Un caso significativo fue el caso de las mujeres de Afganistán. Distintas ONGs y europarlamentarias como Luisa Morgantini y Emma Bonino, impulsaron iniciativas para dar conocer la crudeza de la situación de las mujeres bajo el régimen talibán.

El logro que compendia los esfuerzos de las alianzas forjadas durante años en este sentido es la Resolución 1325 del Consejo de Seguridad. En mayo de 2000, la Liga Internacional de Mujeres por la Paz y la Libertad, junto a Amnistía Internacional, el Llamamiento de La Haya por la Paz, Alerta Internacional, la

28. Ibíd., pp. 88-89.

29. Ibíd., pp. 199-201.

30. Ibíd. 
Asociación Internacional de Investigación para la Paz y la Comisión de Mujeres para Mujeres y niños refugiados, crearon la ONG llamada Grupo de trabajo sobre Mujeres, Paz y Seguridad ${ }^{31}$. Este grupo unió sus esfuerzos a la División para el Avance de las Mujeres (DAW) y el Fondo para el Desarrollo de las Mujeres (UNIFEM) de Naciones Unidas para incidir en los miembros del Consejo de Seguridad con documentación e informes que destacaban y argumentaban la importancia de la presencia de las mujeres en los procesos de paz. La presidencia de Namibia en el Consejo de Seguridad y la implicación de los medios de comunicación abrirían una ventana de oportunidad para que el Consejo acogiera las perspectivas de las mujeres sobre la paz y la guerra.

En octubre de 2000, el Consejo de Seguridad discutió y aprobó la resolución 1325 , en la que exhorta al Secretario General y a los estados miembros a actuar para lograr una mayor inclusión de las mujeres en los procesos de construcción de la paz y de reconstrucción post-conflicto. La Resolución 1325 llama al Consejo de Seguridad, al Secretario General de Naciones Unidas, a los estados miembros y al resto de partes (agencias humanitarias, militares y sociedad civil) a emprender acciones en cuatro áreas distintas que están interrelacionadas: 1) El aumento de la participación de las mujeres en los procesos de paz y la toma de decisiones. 2) El entrenamiento para el mantenimiento de la paz desde una perspectiva de género. 3) La protección de las mujeres en los conflictos armados y en las situaciones post-conflicto y 4) La introducción transversal del género en la corriente principal de recogida de datos y sistemas de información de Naciones Unidas, así como en la puesta en práctica de los programas.

En esta resolución, el Consejo de Seguridad reconoce no sólo que «la paz está inextricablemente unida a la igualdad entre hombres y mujeres» sino que «el acceso pleno y la participación total de las mujeres en las estructuras de poder y su completa implicación en los esfuerzos para la prevención y la resolución de conflictos, son esenciales para el mantenimiento y la promoción de la paz y la seguridad». El doctor Theo-Ben Gurirab, Ministro de Asuntos Exteriores de Namibia y presidente del Consejo de Seguridad, en el momento en que se aprobó la 1325, lo expresaba de este modo: si «las mujeres son la mitad de toda comunidad..., ¿̇no han de ser también la mitad de toda solución?»32.

Este paso bien puede calificarse de histórico, y su significado enlaza con la tradición iniciada por las mujeres en la Primera Guerra Mundial, mencionada antes. Y es que desde hace más de cien años, las mujeres tienen claro que para participar en la toma de decisiones sobre la guerra y la paz, han de unirse a nivel internacional.

Diversos testimonios dan cuenta de la importancia de la línea de acción marcada por la Resolución 1325. El Alto Comisionado de las Naciones Unidas

31. Sobre el Grupo de trabajo sobre Mujeres, Paz y Seguridad (Working Group on Women, Peace and Security), véase la página web: www.peacewomen.org/un/ngo/wg.html

32. Citado en ReHN, E. y Sirleaf, E.J.: Op. cit., p. 76. 
para los Derechos Humanos, posteriormente muerto en Irak, Sergio Vieira de Mello, a comienzos de marzo de 2003, decía:

«En Timor Este pude ver el impacto que la participación de las mujeres puede tener en la construcción de la paz y en el desarrollo (...) Las mujeres son un factor de estabilidad y reconciliación; sus contribuciones pueden mejorar la cualidad de las decisiones y por ende la efectividad de los esfuerzos de recuperación. Sobre todo, promovemos la participación de las mujeres porque tienen el mismo derecho que los hombres a tomar parte en las decisiones y porque su aportación supone un valor añadido en todas las decisiones» ${ }^{33}$.

Además de la inclusión en los procesos de paz, los grupos organizados de mujeres reclaman atención económica, que sus objetivos sean reconocidos como importantes y les sean destinados fondos, algo que no suele suceder al considerar sus preocupaciones de importancia menor. Como dice Jamila, de Afganistán:

"Oigo frecuentemente decir que las afganas no somos políticas. Que la paz y la seguridad son asuntos de hombres. Estoy aquí para rebatir esa ilusión. En los 20 últimos años de mi vida, el liderazgo de los hombres sólo nos ha traído guerra y sufrimiento. Soy una mujer que lucha por la educación. En Afganistán y Pakistán esto es política. Dirijo una ONG humanitaria. En Afganistán y Pakistán esto es política (...) Vemos que se canaliza la financiación hacia organizaciones de hombres afganos y me pregunto por qué...» ${ }^{34}$.

La tesis del Grupo de trabajo sobre Mujeres, Paz y Seguridad, es que la desigualdad de género es una amenaza para la paz y la seguridad globales. Para confrontar esta amenaza proponen que se tengan en cuenta las necesidades y capacidades de hombres y mujeres, que la perspectiva de género oriente la actuación en todas las fases de un conflicto armado: en la prevención, en la negociación y en la protección de los civiles. El grupo que consiguió la aprobación de la Resolución 132 está ahora trabajando por su extensión, dándola a conocer y presionando para que esta importante herramienta política dirigida a la inclusión de las mujeres en los procesos de paz, se aplique.

\section{BIBLIOGRAFÍA}

Alonso Ollacarizoueta, Lucía: Reanudar lazos rotos. Un estudio sobre reconciliación en Camboya, Barcelona, Icaria, 2003.

Anderlini, Sanam Naraghi: Women at the Peace Table. Making a Difference, NuevaYork, The United Nations Development Fund for Women (UNIFEM), 2000.

BARNES, Catherine (ed.): Haciendo propio el proceso. La participación ciudadana en los procesos de paz, Gernika-Lumo, Gernika Gogoratuz, 2004.

Bogodo-Madikizela, Pumla: Women's Contributions to South Africa's Truth and Reconciliation Commission, Women Waging Peace, The Hunt Fund, 2005.

33. Intervención realizada en la Comisión de Naciones Unidas sobre el estatus de las mujeres, Nueva York, 7 de marzo de 2003.

34. Intervención en el Consejo de Seguridad, octubre de 2001. 
Brock-Utne, Birgit: Educating for Peace. A Feminist Perspective, Oxford, Pergamon Press, 1985.

BUSSEY, Gertrude y TIMS, Margaret: Pioneers for Peace. Women's International League for Peace and Freedom 1915-1965, Oxford, Alden Press, 1980.

ELSHTAIN, Jean Bethke: Women and War, Chicago, The University of Chicago Press, 1995.

FARHAT-NASER, Sumaya: En la tierra de los olivos. Una historia de mujeres por la paz (Prólogo y traducción de Anna Tortajada), Barcelona, El Aleph, 2006.

GraU BiosCA, Elena: «1980-2005, veinticinco años sin Giulia y con Giulia», Mientras Tanto, 94 (2005), pp. 39-44.

- «Sentada en mi lado del abismo. Sobre Tres Guineas de Virginia Woolf», En Pie de Paz, 52 (2000), pp. 40-47.

GÓMEZ, Lourdes: «Mo Mowlam, la ministra británica que consolidó la paz en Irlanda del Norte", El País, sábado 20 de agosto de 2005, p. 45.

LIDDINGTON, Jill: «La campaña de las mujeres por la paz. Historia de una lucha olvidada», en Dorothy Thompson (comp.): Over our dead bodies, Londres, Virago, 1983 (Trad. Mireia Bofill, Antes muertas. Mujeres contra el peligro nuclear, Barcelona, LaSal, 1984, pp. 192-210).

MAGAlLón PORTOLÉs, Carmen: Mujeres en pie de paz. Madrid, Siglo XXI, 2006.

- «El derecho humano a la paz y la sociedad civil. Una mirada desde las vidas de las mujeres», en Xesús R. Jares, Josu Ugarte, Mikel Mancisidor y Maria Oianguren (coords.): El papel de la investigación para la paz ante la violencia en el País Vasco, Bilbao, Bakeaz y Gernika Gogoratuz, 2006, pp. 117-136.

- "Las mujeres como sujeto colectivo de construcción de paz", Bilbao, Bakeaz, Escuela de Paz, 2004.

MOWLAN, Marjorie: "Combustible para la carrera del armamento nuclear. Energía nuclear y armas nucleares", en Dorothy Thompson (comp.): Over our dead bodies, Londres, Virago, 1983 (Trad. Mireia Bofill, Antes muertas. Mujeres contra el peligro nuclear, Barcelona, LaSal, 1984, pp. 93-103).

MURARO, Luisa: «Si la política vence a la guerra», L'Unitá, viernes 4 de junio de 1999.

NASH, Mary: Mujeres en el mundo. Historia, retos y movimientos, Madrid, Alianza, 2004.

OlDFIELD, Sybil: Women Against the Iron Fit. Alternatives to Militarism (1900-1989), Cambridge, Basil Blackwell, 1989.

ReHN, Elizabeth y SiRLEAF, Ellen J.: Women War and Peace. The Independent Experts'Assesment on the Impact of Armed Conflict on Women and Women's Role in Peace-building, Nueva York, The United Nations Development Fund for Women (UNIFEM), 2002.

SHIVA, Vandana: Abrazar la vida. Mujer, ecología y supervivencia (Traducción de Ana Elena Guyer y Beatriz Sosa), Madrid, Horas y horas, 1995.

VeLLACOT, Jo: «A Place for Pacifism and Transnationalism in Feminist Theory: the early work of the Women's International League for Peace and Freedom", Women's History Review, 2:1 (1993), pp. 23-56.

Woolf, Virginia: Tres Guineas, Barcelona, Lumen, 1977 (1938). 